\title{
Physical optics modeling of Sky coverage for TMT NFIRAOS with advanced LQG controller
}

\author{
Lianqi Wang $^{a}$, Luc Gilles ${ }^{a}$, Brent Ellerbroek ${ }^{a}$, and Carlos Correia ${ }^{b}$ \\ ${ }^{a}$ Thirty Meter Telescope Project, 1111 S. Arroyo Pkwy, Suite 200, Pasadena, CA, 91105, USA \\ ${ }^{b}$ Centre for Astrophysics, University of Porto, Rua das Estrelas, 4150-762 Porto, Portugal \\ Send correspondence to Lianqi Wang (lianqiw@tmt.org)
}

\begin{abstract}
We have implemented the linear quadratic Gaussian (LQG) controller in our physical optics sky coverage simulator (MAOS) for the Thirty Meter Telescope (TMT) Narrow Field InFrared Adaptive Optics System (NFIRAOS) aimed for improved correction of tip/tilt and plate scale modes. The LQG controller has a built-in capability to correct narrow frequency vibrations that are above the closed loop bandwidth of the system and is a very desirable solution for this application. The LQG controller is tuned with the combined power spectral density (PSD) of turbulence, wind shake, and vibration computed from the telemetry. We will show how LQG performs for various telescope/instrument vibration spectral (such as broadband or drifting peaks). We will also show the performance and sky coverage of LQG in comparison with single or double integrator controllers for correcting low order atmospheric turbulence with a set of up to three tip/tilt(/focus) natural guide star wavefront sensors. We found that the LQG controller reduces the median sky coverage wavefront error by $25 \mathrm{~nm}$ in quadrature.
\end{abstract}

\section{INTRODUCTION}

The Linear Quadratic Gaussian (LQG) controller is a combination of linear quadratic control and Kalman filtering. The linear quadratic control requires full state information and uses a state feedback controller determined by Riccati recursion, while the Kalman filter is a technique to estimate the state from measurement of the output.

We implemented the LQG controller to control modes from measurements of on-instrument natural guide star wavefront sensors (OIWFS). In addition to improved rejection of tip/tilt caused by turbulence and/or telescope wind shake, the LQG controller is also capable of suppressing vibrations beyond the closed loop bandwidth when the controller is tuned with a prior knowledge of the vibration lines. The Type I (single integrator) or Type II (double integrators in series with a lead filter) controllers, on the other hand, have to be paired with other algorithms to suppress vibration. ${ }^{1}$

Simulations with simplified turbulence model has been presented before showing that using LQG controller improves sky coverage in LGS MCAO systems. ${ }^{2}$ In this paper, we present our slightly different formulation and performance results in an end-to-end, physical optics modeling of the sky coverage.

\subsection{Model Identification}

In the continuous time domain, the contribution of turbulence, wind shake, or vibration to any OIWFS mode $\mu_{i}(t)$ is approximately represented by a second order stochastic differential equation (SDE) driven by Gaussian white noise:

$$
\ddot{\mu}(t)+c_{1} \dot{\mu}(t)+c_{2} \mu(t)=\xi(t)
$$

where, for simplicity, we have omitted the subscript $i$. Applying a Fourier transform to both side, we get:

$$
\frac{\hat{\mu}(f)}{\hat{\xi}(f)}=\frac{1}{s^{2}+c_{1} s+c_{2}}=\frac{1}{-\omega^{2}+i c_{1} \omega+c_{2}} .
$$


with $s=i \omega=2 \pi i f$. The PSD of $\mu(t)$ can be computed from its Fourier transform by

$$
\begin{aligned}
\Phi_{\mu}(f) & =\int\left\langle\hat{\mu}(f) \hat{\mu}\left(f^{\prime}\right)\right\rangle \mathrm{d} f^{\prime} \\
& =\frac{\sigma_{\xi}^{2}}{\left(c_{2}-\omega^{2}\right)^{2}+c_{1}^{2} \omega^{2}}
\end{aligned}
$$

where we have used $\left\langle\xi(f) \xi\left(f^{\prime}\right)\right\rangle=\sigma_{\xi}^{2} \delta\left(f-f^{\prime}\right)$ because $\xi$ is a stationary Gaussian random variable. For a vibration system, usually $c_{2}$ is the angular resonance frequency squared and $c_{1}$ is a function of the damping ratio.

The covariance of $\mu(t)$ equals to the inverse Fourier transform of its 2-sided PSD. This covariance function can then be fitted to the covariance of the OIWFS modes using $N$ leading points to determine $c_{1}, c_{2}$, and $\sigma_{\xi}$. In our modeling, we used $\mathrm{N}$ corresponding to $\sim 0.5$ second in time. Alternatively, PSD fitting may be used that directly fits Eq 4 to the PSD of the OIWFS mode, with optional weighting to select the frequency range of interest. ${ }^{3}$ The SDE parameters for each OIWFS mode is obtained independently.

\subsection{Continuous time state space vector}

This SDE can be rewritten in state space model by introducing a state space vector

$$
\mathbf{x}(t)=\left(\begin{array}{c}
\dot{\mu}(t) \\
\mu(t)
\end{array}\right)
$$

which obeys:

$$
\begin{aligned}
\dot{\mathbf{x}}(t) & =\left(\begin{array}{cc}
-c_{1} & -c_{2} \\
1 & 0
\end{array}\right) \mathbf{x}(t)+\left(\begin{array}{l}
\xi \\
0
\end{array}\right)(t) \\
& \equiv A \mathbf{x}(t)+\boldsymbol{\xi}(t)
\end{aligned}
$$

where $c_{1}, c_{2}$ and $\xi$ are parameters identified in the previous section. The Gaussian white noise vector $\boldsymbol{\xi}$ has a covariance of

$$
\Sigma_{\boldsymbol{\xi}}=\left\langle\boldsymbol{\xi} \boldsymbol{\xi}^{T}\right\rangle=\left(\begin{array}{cc}
\sigma_{\xi}^{2} & 0 \\
0 & 0
\end{array}\right)
$$

The solution of the state from initial condition can be written as

$$
\mathbf{x}(t)=e^{A\left(t-t_{0}\right)} \mathbf{x}\left(t_{0}\right)+\int_{t_{0}}^{t} e^{A(t-\tau)} \boldsymbol{\xi}(\tau) \mathrm{d} \tau
$$

where $e^{A t}$ is the state transition matrix* of the system, $t_{0}$ and $\mathbf{x}\left(t_{0}\right)$ are the initial time and state.

Certain PSDs may need two or more different set of SDE parameters, like the turbulence plus telescope vibration. In this case, we would find two (or more) set of SDE parameters $\left(c_{1}, c_{2}\right.$, and $\left.\xi\right)$ and concatenate the state vector from each set. The state evolution operator $A$ will be a block diagonal matrix.

\subsection{Discrete time state space vector}

To build the digital controller, we have to digitize the continuous time state space vector. Define the discrete state space model as $\mu_{k}=\mu(k T)$ and $\mathbf{x}_{k}=\mathbf{x}(k T)$ :

$$
\mathbf{x}_{k}=\left(\begin{array}{c}
\dot{\mu}_{k} \\
\mu_{k}
\end{array}\right)
$$

\footnotetext{
${ }^{*}$ Note: the matrix exponential can be computed using a simple scaling and squaring method. First divide the matrix by $2^{N}$ so that it is close to zero. Compute its exponential using Taylor expansion with around 10 terms, then do a $2^{N}$ th power on the result to get the answer.
} 
Following equation 9 , and set $t_{0}$ to $k T$, we can obtain the state evolution model:

$$
\begin{aligned}
\mathbf{x}_{k+1} & =e^{A T} \mathbf{x}_{k}+\int_{k T}^{(k+1) T} e^{A((k+1) T-\tau)} \boldsymbol{\xi}(\tau) \mathrm{d} \tau \\
& \equiv e^{A T} \mathbf{x}_{k}+\boldsymbol{\epsilon}_{k}
\end{aligned}
$$

The covariance of the noise term, $\boldsymbol{\epsilon}_{k}$, is

$$
\begin{aligned}
\Sigma_{\boldsymbol{\epsilon}} & =\int_{k T}^{(k+1) T} \int_{k T}^{(k+1) T} e^{A((k+1) T-\tau)} \Sigma_{\boldsymbol{\xi}} e^{A^{T}\left((k+1) T-\tau^{\prime}\right)} \delta\left(\tau-\tau^{\prime}\right) \mathrm{d} \tau \mathrm{d} \tau^{\prime} \\
& =\int_{0}^{T} e^{A t} \Sigma_{\boldsymbol{\xi}} e^{A^{T} t} \mathrm{~d} t
\end{aligned}
$$

which can be evaluated numerically or analytically.

The average of the state over period of $T$ is :

$$
\overline{\mathbf{x}}_{k}=\frac{1}{T} \int_{(k-1) T}^{k T} \mathbf{x}(t) \mathrm{d} t .
$$

Again following equation 9 , and set $t_{0}$ to $k T$, we have

$$
\begin{aligned}
\overline{\mathbf{x}}_{k} & =\frac{1}{T} \int_{(k-1) T}^{k T}\left(\left(e^{A(t-k T)} \mathbf{x}_{k}+\int_{k T}^{t} e^{A(t-\tau)} \boldsymbol{\xi}(\tau) \mathrm{d} \tau\right) \mathrm{d} t\right. \\
& =\frac{1}{T}\left(I-e^{-A T}\right) A^{-1} \mathbf{x}_{k}-\frac{1}{T} \int_{(k-1) T}^{k T}\left(\int_{(k-1) T}^{\tau} e^{A(t-\tau)} \mathrm{d} t\right) \boldsymbol{\xi}(\tau) \mathrm{d} \tau \\
& =\frac{1}{T}\left(I-e^{-A T}\right) A^{-1} \mathbf{x}_{k}-\frac{1}{T} \int_{0}^{T}\left(I-e^{-A \tau}\right) A^{-1} \boldsymbol{\xi}(\tau) \mathrm{d} \tau \\
& \equiv Q \mathbf{x}_{k}+\zeta
\end{aligned}
$$

with the state averaging operator

$$
Q=\frac{1}{T}\left(I-e^{-A T}\right) A^{-1}
$$

and a noise term $\zeta$ with covariance of

$$
\Sigma_{\boldsymbol{\zeta}}=\frac{1}{T^{2}} \int_{0}^{T}\left(I-e^{-A t}\right) A^{-1} \Sigma_{\boldsymbol{\xi}} A^{-T}\left(I-e^{-A^{T} t}\right) \mathrm{d} t
$$

which again can be evaluated numerically.

The average of the OIWFS mode over a sampling period is selected from the state space model:

$$
\bar{\mu}_{k}=P \overline{\mathbf{x}}_{k}=P Q \mathbf{x}_{k}+P \zeta
$$

where $P=\left[\begin{array}{ll}0 & 1\end{array}\right]$ selects the second row of $\overline{\mathbf{x}}$.

\subsection{Kalman Filter and LQG}

To build the Kalman filter using the quantities described in the previous section, we define the discrete state space as a concatenated vector from each OIWFS or vibration mode:

$$
\boldsymbol{x}_{k}=\left(\begin{array}{c}
\mathbf{x}_{k}^{0} \\
\vdots \\
\mathbf{x}_{k}^{n}
\end{array}\right) \equiv\left[\mathbf{x}_{k}\right]_{c}
$$


where we have used []$_{c}$ to denote column concatenation of vectors. The full state space model of all OIWFS modes and the measurement by OIWFS can be written as:

$$
\begin{aligned}
\boldsymbol{x}_{k+1} & =\mathcal{A} \boldsymbol{x}_{k}+\boldsymbol{\epsilon}_{k} \\
\boldsymbol{s}_{k} & =\mathcal{C} \boldsymbol{x}_{k}-\mathcal{D} \boldsymbol{u}_{k-1}+\boldsymbol{\eta}_{k}
\end{aligned}
$$

where the state transition matrix $\mathcal{A}$ and the state noise covariance $\Sigma_{\boldsymbol{\epsilon}}$ are block diagonal concatenation of the corresponding quantities for each OIWFS mode:

$$
\begin{aligned}
\mathcal{A} & =\left(\begin{array}{ccc}
e^{A_{0} T} & & \\
& \ddots & \\
& & e^{A_{n} T}
\end{array}\right) \equiv\left[e^{A T}\right]_{d} \\
\Sigma_{\boldsymbol{\epsilon}} & =\left[\Sigma_{\epsilon}\right]_{d}
\end{aligned}
$$

where we have used []$_{d}$ to denote diagonal concatenation of square matrices. Each OIWFS measurement $\boldsymbol{s}_{k}$ is from the average of all the OIWFS modes minus the deformable mirror (DM) correction (if closed loop) over a sampling period plus some measurement noise. The gradient operator $\mathcal{C}$ and noise covariance matrix $\Sigma_{\boldsymbol{\eta}}$ take the form:

$$
\begin{aligned}
\mathcal{C} & =[G]_{c}[P Q]_{d} \\
\Sigma_{\boldsymbol{\eta}} & =\left[\Sigma_{\eta}\right]_{d}
\end{aligned}
$$

where $G$ is the OIWFS modes to gradient interaction matrix for each OIWFS. The measurement noise of each OIWFS has a covariance of $\Sigma_{\eta}=G\left[P \Sigma_{\zeta} P\right]_{d} G^{T}+\Sigma_{n}$, where the first quantity is due to the state noise and $\Sigma_{n}$ is due to the photon and detector readout noise.

Here the WFS measurement $\boldsymbol{s}_{k}$ is related to the state space mode of the turbulence at step $k, \boldsymbol{x}_{k}$ and DM correction at step $k-1$ because it is a discrete command applied at step $k-1$ with its effect only showing up at step $k$ due to OIWFS integration. The DM to WFS gradient operator $\mathcal{D}$ will be set to zero when working with pseudo open loop gradients with fast DM. For DMs with finite bandwidth, $\mathcal{D}$ will take that into account.

The Kalman filter is obtained from an estimation of the recursive Riccati equation in steady state. ${ }^{4}$ The asymptotic Kalman Gain $\mathcal{L}_{\infty}$ is computed as

$$
\mathcal{L}_{\infty}=\mathcal{A H} \mathcal{H}_{\infty}=\mathcal{A} \Sigma_{\infty} \mathcal{C}^{T}\left(\mathcal{C} \Sigma_{\infty} \mathcal{C}^{T}+\Sigma_{\eta}\right)^{-1}
$$

with the estimation error covariance matrix $\Sigma_{\infty}$ as the solution of the algebraic Riccati equation:

$$
\Sigma_{\infty}=\mathcal{A} \Sigma_{\infty} \mathcal{A}^{T}+\Sigma_{\boldsymbol{\epsilon}}-\mathcal{A} \Sigma_{\infty} \mathcal{C}^{T}\left(\mathcal{C} \Sigma_{\infty} \mathcal{C}^{T}+\Sigma_{\boldsymbol{\eta}}\right)^{-1} \mathcal{C} \Sigma_{\infty} \mathcal{A}^{T}
$$

Here $\Sigma_{\boldsymbol{\epsilon}}$ and $\Sigma_{\boldsymbol{\eta}}$ are the covariance matrices of the state noise and measurement noise as defined above. The matrix inversion should be done with truncated SVD so that poorly sensed modes will not pollute the estimation.

Finally, we obtain the estimation of the state at $k+1$ with the LQG controller,

$$
\hat{\boldsymbol{x}}_{k+1 \mid k}=\mathcal{A} \hat{\boldsymbol{x}}_{k \mid k-1}+\mathcal{L}_{\infty}\left(\boldsymbol{s}_{k}-\left(\mathcal{C} \hat{\boldsymbol{x}}_{k \mid k-1}-\mathcal{D} \boldsymbol{u}_{k-1}\right)\right)
$$

For a general AO system with two cycle delay, if the OIWFS sampling rate is the same as the main AO system (determined by the high order WFS), an additional $\mathcal{A}$ need to be applied to predict the estimate further into the future (step $k+2$ ) and then compute the DM command to be applied at step $k+1$ from the average of the mode from step $k+1$ to $k+2$ :

$$
\boldsymbol{u}_{k+1}=F[P Q]_{d} \hat{\boldsymbol{x}}_{k+2 \mid k}=F[P Q]_{d} \mathcal{A} \hat{\boldsymbol{x}}_{k+1 \mid k}
$$




\begin{tabular}{|c|c|}
\hline Vibration & RMS WFE (nm) \\
\hline \hline Input & 250 \\
\hline LQG & 9.6 \\
\hline Type I & 250 \\
\hline Type II & 250 \\
\hline
\end{tabular}

Table 1. Input and Residual of vibration control simulation with noise free OIWFS at $80 \mathrm{~Hz}$

where $F$ projects the averaged OIWFS mode onto DM actuator command. For dim OIWFS stars, the OIWFS loop may be running at a reduced rate than the main $\mathrm{AO}$ system. Let $M$ be the ratio between the sampling frequency of the OIWFS loop and the main AO system, we will predict the estimate and compute DM commands at the rate of the main $\mathrm{AO}$ system, i.e., up-sampling the control signal:

$$
u_{k+j / M}=F\left[P Q_{M}\right]_{d} \mathcal{A}_{M}^{j} \hat{x}_{k+1 \mid k} j=1 \ldots M
$$

which falls back to Eq 33 when $M=1$. Here the state transition matrix $\mathcal{A}_{M}^{j}$ and averaging operator $Q_{M}$ for each OIWFS mode are defined at the AO system main frequency

$$
\begin{aligned}
\mathcal{A}_{M}^{j} & =\left[e^{A T(j / M)}\right]_{d} \\
Q_{M} & =\frac{M}{T}\left(I-e^{-A T / M}\right) A^{-1} .
\end{aligned}
$$

We compared the LQG controller against Type I and II controllers are presented the results in the following sections.

\section{VIBRATION SUPPRESSION}

In the simulations described in this section, we employed a single geometric, noise free $\mathrm{T} / \mathrm{T}$ WFS measuring at $80 \mathrm{~Hz}$. We assumed the vibration power spectrum density (PSD) function was known. Without loss of generality, we also assumed there was only one vibration mode with certain FWHM around the peak frequency in the PSD.

We first considered a vibration PSD with $29 \mathrm{~Hz}$ peak frequency and $0.5 \mathrm{~Hz}$ FWHM. We fitted the covariance function of the 2nd order SDE model to this vibration mode for up to 0.5 second duration. Figure 1 shows the fitting of the SDE model to the vibration PSD. The SDE model contains more lower and higher frequency contents than the vibration PSD due to mismatch between the models. Table 1 shows the root mean square (RMS) residual wavefront error (WFE) with LQG control and the Type I (single integrator) and Type II controller (two integrators in series). The LQG suppressed the vibration to less than $4 \%$ RMS while the Type I and Type II controller cannot achieve any suppression because the vibration frequency at $29 \mathrm{~Hz}$ is way above the control bandwidth of these two controllers.

We then varied the vibration frequency and/or FWHM while keeping the WFS at $80 \mathrm{~Hz}$. The LQG controller was always tuned to the real vibration PSD. Figure 2 shows the residual error as a fraction of the RMS input. We can see that, when the FWHM is less than $0.6 \mathrm{~Hz}$, and the vibration frequency is a few $\mathrm{Hz}$ away from harmonics of the WFS Nyquist frequency $(40 \mathrm{~Hz})$, the LQG controller can reduce the vibration error to below $5 \%$ RMS of the input. The residual is roughly proportional to the FWHM. When the FWHM is larger than $1 \mathrm{~Hz}$, the residual become worse than than $7.5 \%$.

Next we kept the LQG controller tuned to $32 \mathrm{~Hz}$ vibration, but vary the actual vibration frequency to study the robustness of the LQG controller. Figure 3 shows the residual as a function of the actual vibration frequency. The residual becomes worse than $10 \%$ when the vibration has drifted by about $0.5 \mathrm{~Hz}$ when the FWHM is no more than $0.5 \mathrm{~Hz}$. 



Figure 1. SDE fitting for $29 \mathrm{~Hz}$ vibration with $0.5 \mathrm{~Hz}$ FWHM.

\section{TURBULENCE REJECTION}

We compared the performance of LQG control against Type I and Type II controller for a tip/tilt PSD of turbulence and telescope wind shake. Figure 4 shows the fitting of the SDE model to the tip/tilt PSD. While the agreement in the covariance function is fairly well for $\leq 1$ second separations, there is a mismatch in the PSD at high frequency.

The WFS sampling frequency was varied from 20 to $800 \mathrm{~Hz}$ and the measurement noise was varied from 0 to 5 milli-arcseconds (mas). Figure 5 shows the RMS residual of the tip/tilt modes after correction by LQG (solid lines), Type I (dotted) or Type II (dashed) controllers for each noise level. When the WFS sampling frequency is less than $100 \mathrm{~Hz}$, the LQG controller clearly enjoys a performance advantage against the type II controller, which is again better than Type I controller. When the WFS sampling frequency is higher than $100 \mathrm{~Hz}$, the LQG out performs both Type I and Type II controllers when noise is high, but under performs when noise is low, primarily due to model mismatch (See Figure 4).

\section{SKY COVERAGE}

NFIRAOS is an MCAO system with two deformable mirrors conjugated to the range of 0 and $11.3 \mathrm{~km}$ respectively, an asterism of 6 sodium laser guide stars arranged in a pentagon with 35" radius plus one more on axis, and up to three natural guide star on-instrument low-order wavefront sensors (OIWFS). At least one of the OIWFS will employ $2 \times 2$ lenslet array to provide focus measurement to compensate for the range variation of the sodium layers.

The performance requirement for NFIRAOS include diffraction limited turbulence compensation over fields of view of up to 30" in diameter and better than 2 mas residual in tip/tilt modes at median sky coverage along Galactic Pole. The sky coverage is limited by the availability of natural guide stars (three required for good correction of tilt-anisoplanatism modes) that are bright and close enough to science target to provide adequate measurement to compensate for tip/tilt and tilt-anisoplanatism modes caused by 1) atmospheric turbulence, 2) 

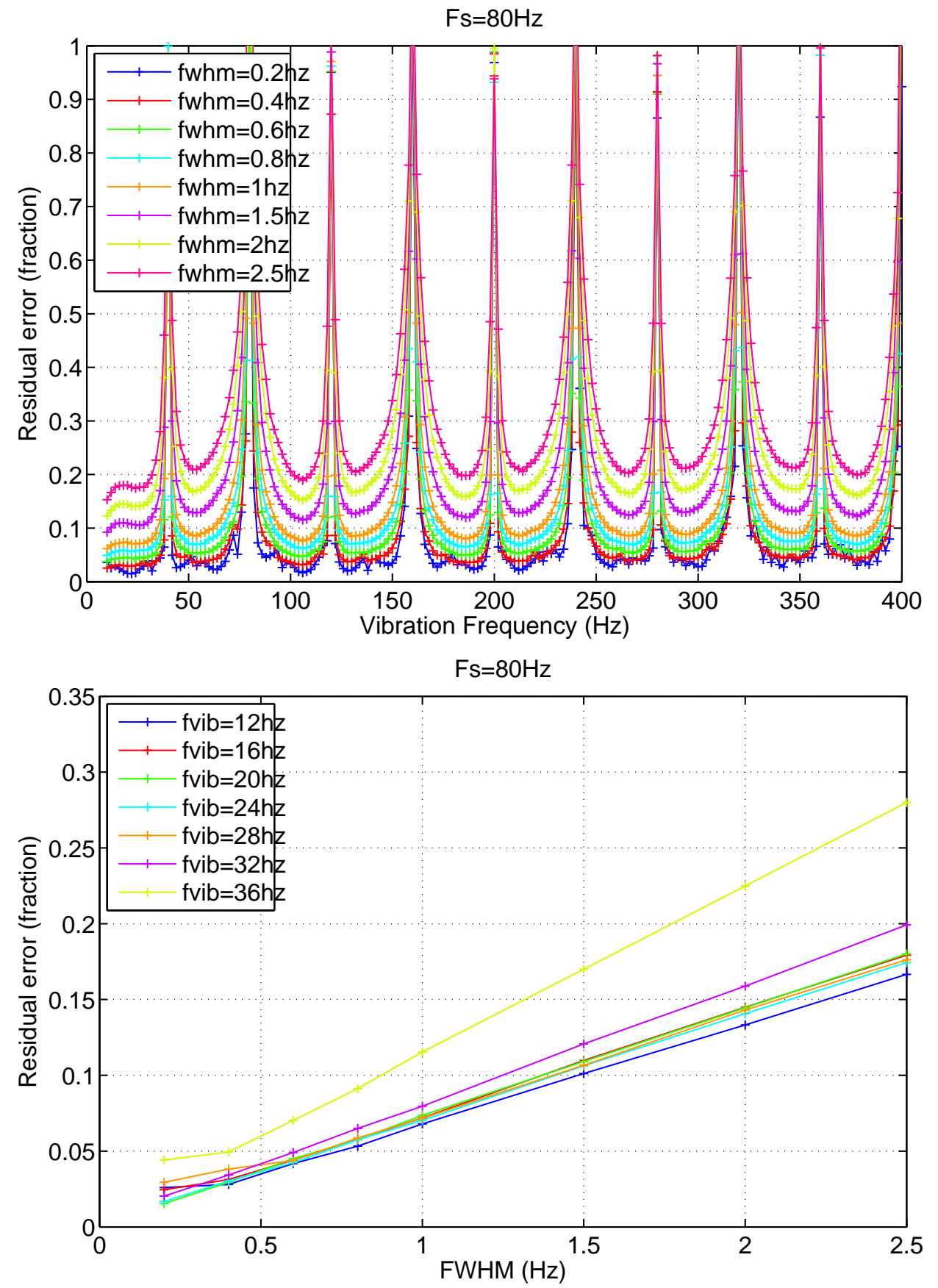

Figure 2. Vibration rejection residual as a function of vibration frequency and FWHM. 


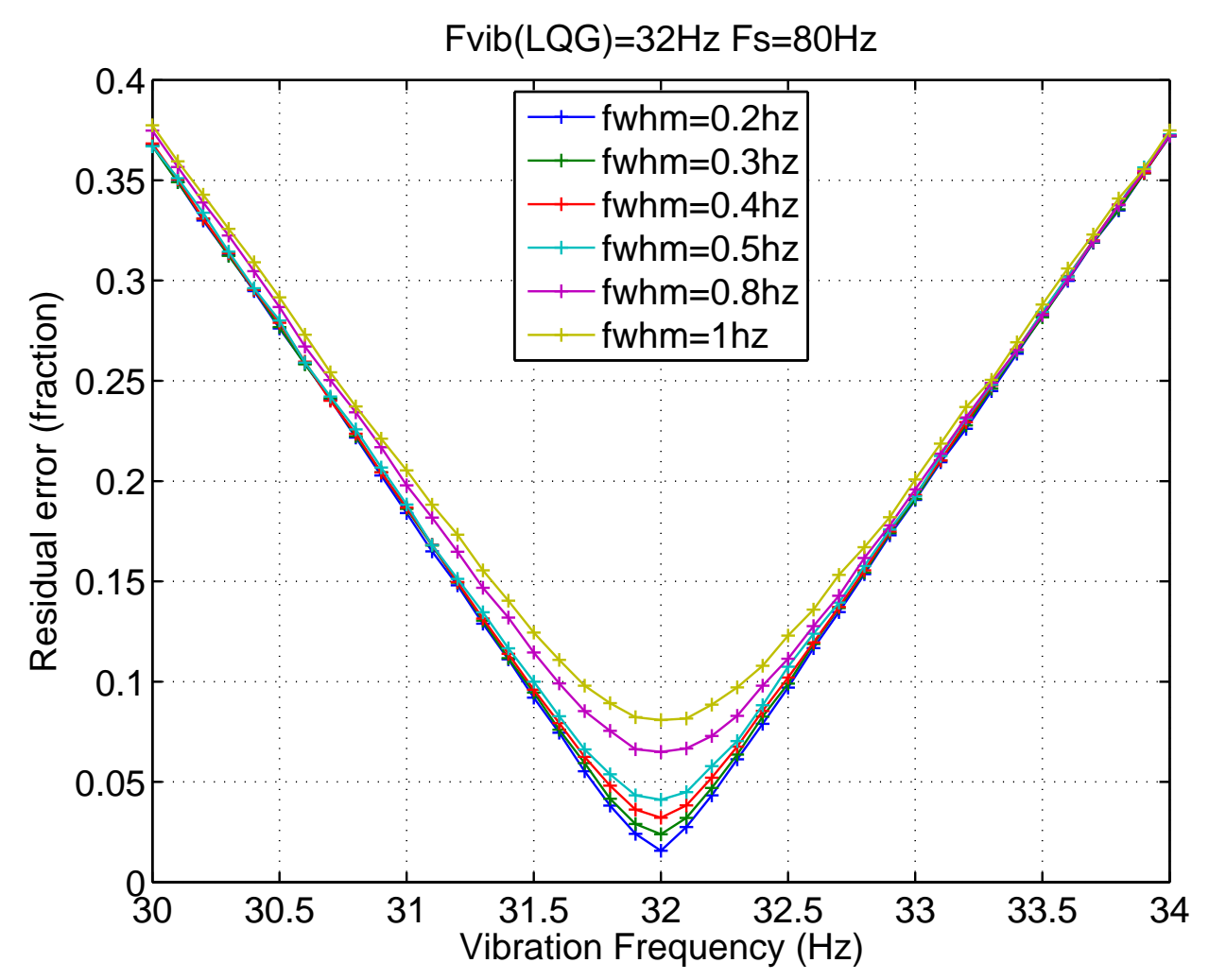

Figure 3. Vibration rejection residual with drifting vibration.
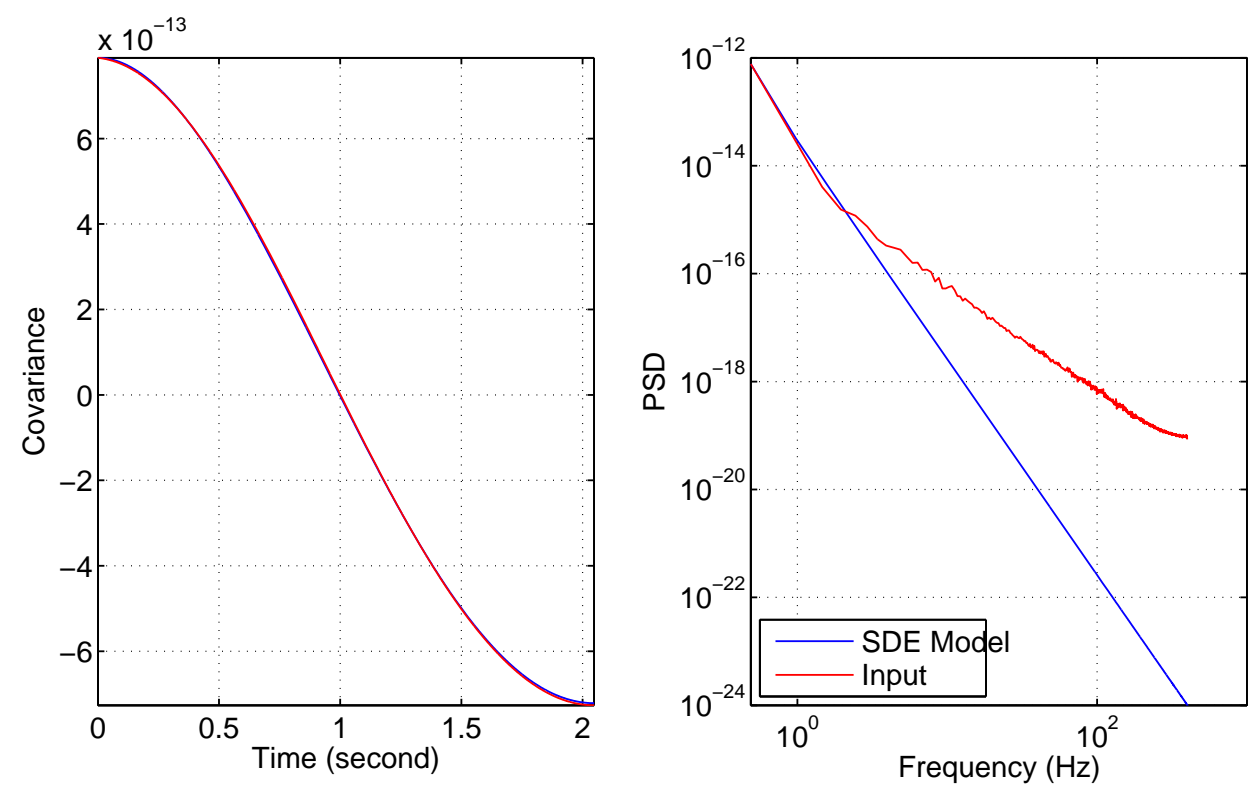

Figure 4. SDE fitting for a tip/tilt PSD of turbulence and telescope wind shake. 


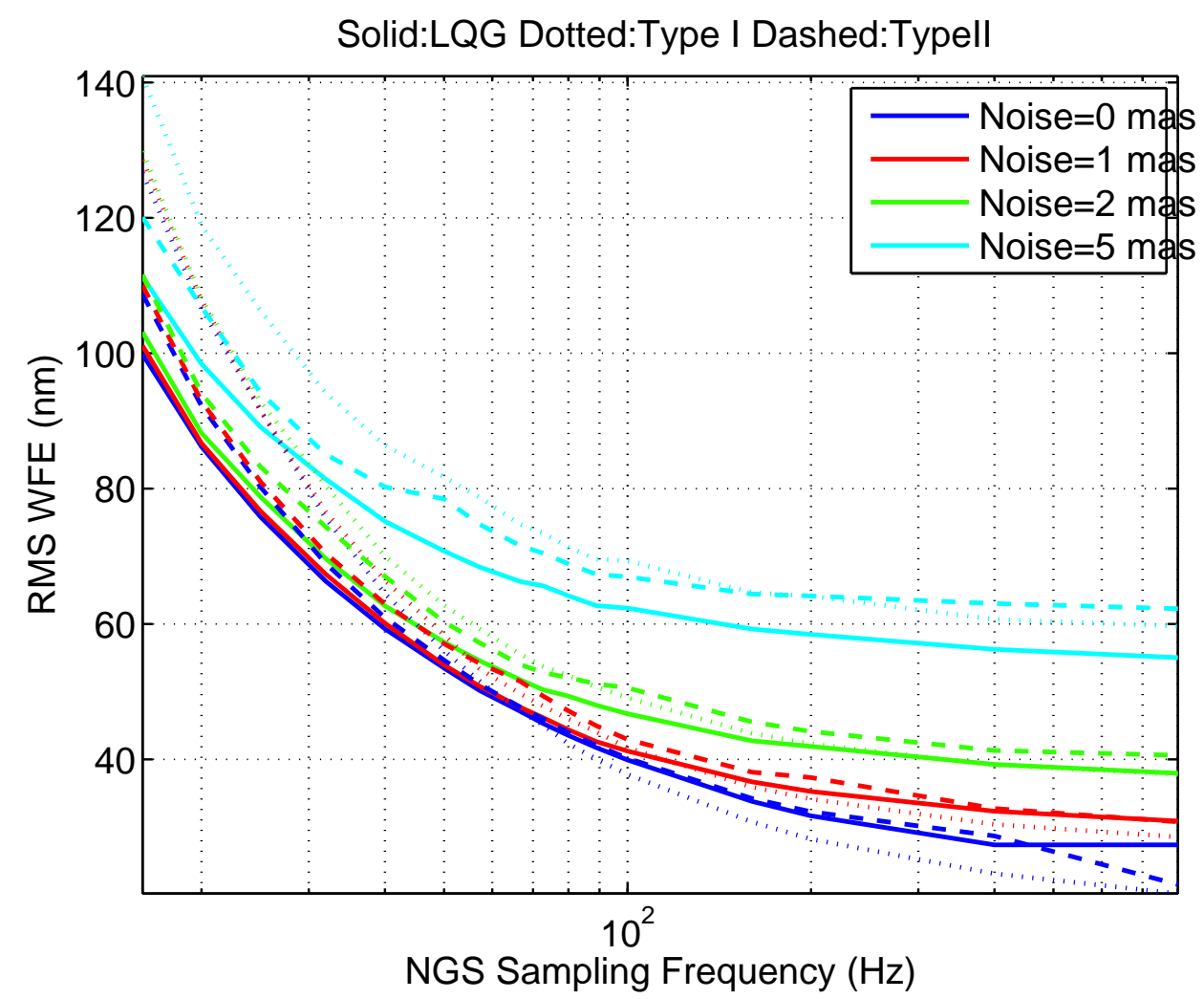

Figure 5. Correction residual for turbulence tip/tilt PSD for LQG, Type I and II controllers.

telescope wind shake, and 3) mechanical vibration. While tip/tilt of turbulence and wind shake often have low bandwidth, the mechanical vibration usually have frequencies in the range of 30-100 Hz. The OIWFS often have to run at low sampling frequencies $(\lesssim 100 \mathrm{~Hz})$ to achieve enough signal to noise ratio. Therefore, a controller that can achieve enough rejection at low sampling frequencies while in the mean time provide enough vibration suppression at high frequencies are highly desired.

We carried out end to end physical optics modeling of the sky coverage for NFIRAOS with the Type I, II, and LQG controllers to compare their performance. The telescope is at zenith while the natural guide stars are selected based on the model of Galactic Pole. ${ }^{5}$ Turbulence and telescope wind shake are included in the model, while mechanical vibration has not been included yet. Please see ${ }^{6}$ for detailed description of NFIRAOS and the simulation method. Figure 6 shows the results. The Type I and Type II controller are almost neck to neck, with RMS WFE of 68.3 and $68.8 \mathrm{~nm}$ at median sky coverage respectively. The LQG controller clearly enjoys a performance advantage, with RMS WFE of $63.6 \mathrm{~nm}$ at median sky coverage, which represents a $25 \mathrm{~nm}$ reduction in RMS WFE quadrature.

Combined with its built-in ability to reject mechanical vibration at high frequencies, the LQG controller seems to be the preferred choice for controlling OIWFS modes. Our next step is to model the sky coverage in the presence of simulated mechanical vibration.

\section{CONCLUSION}

We have presented our formulation of the LQG controller for controlling tip/tilt and tilt-anisoplanatism modes. and showed that the LQG controller can suppress vibration lines higher than closed loop bandwidth while in the same time achieves better rejection of low order modes from atmospheric turbulence and telescope wind shake. 


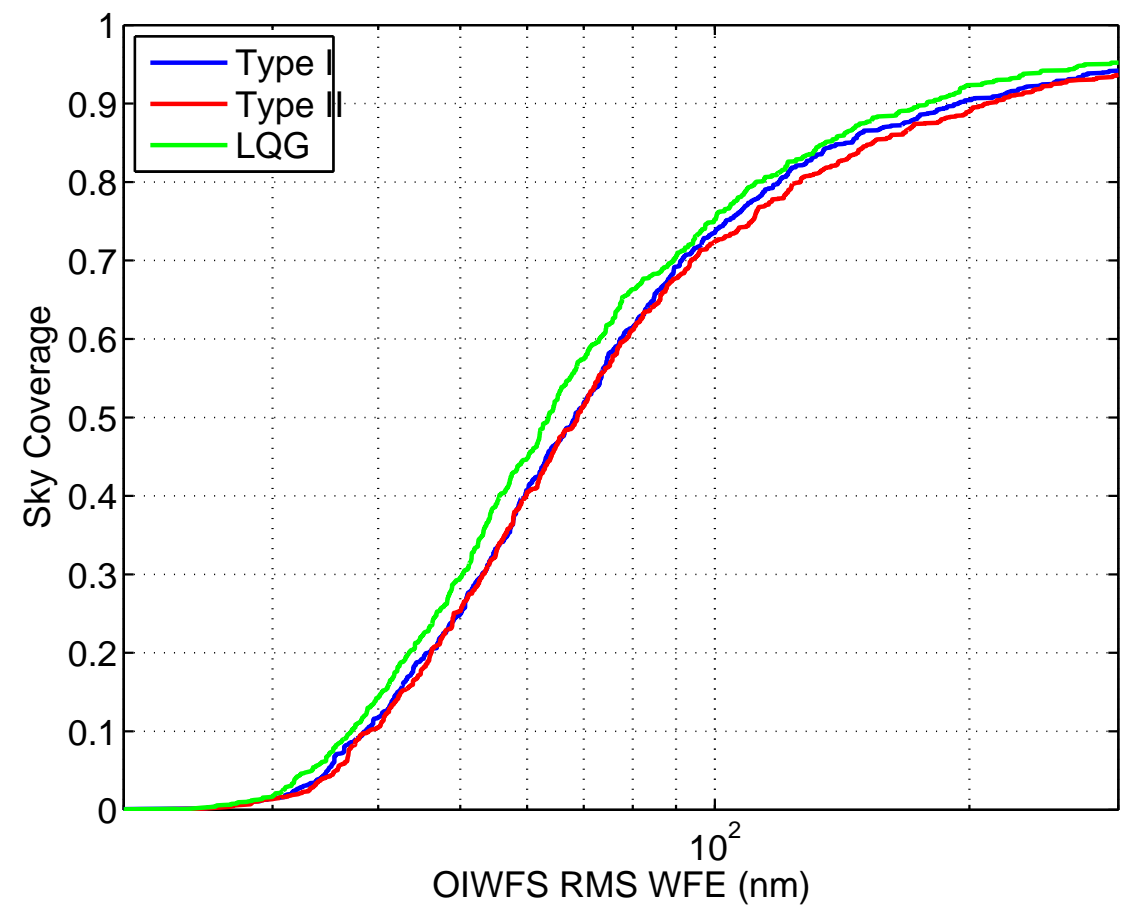

Figure 6. Sky coverage at Galactic Pole for Type I, II, and LQG controllers.

\section{Acknowledgment}

The TMT Project gratefully acknowledges the support of the TMT collaborating institutions. They are the Association of Canadian Universities for Research in Astronomy (ACURA), the California Institute of Technology, the University of California, the National Astronomical Observatory of Japan, the National Astronomical Observatories of China and their consortium partners, and the Department of Science and Technology of India and their supported institutes. This work was supported as well by the Gordon and Betty Moore Foundation, the Canada Foundation for Innovation, the Ontario Ministry of Research and Innovation, the National Research Council of Canada, the Natural Sciences and Engineering Research Council of Canada, the British Columbia Knowledge Development Fund, the Association of Universities for Research in Astronomy (AURA), the U.S. National Science Foundation and the National Institutes of Natural Sciences of Japan.

C. Correia acknowledges the support of the European Research Council through the Marie Curie IntraEuropean Fellowship with reference FP7-PEOPLE-2011-IEF, number 300162.

\section{REFERENCES}

[1] Muradore, R., Pettazzi, L., Fedrigo, E., and Clare, R., "On the rejection of vibrations in Adaptive Optics Systems," SPIE 8447, 38 (2012).

[2] Correia, C., Véran, J.-P., and Herriot, G., "Advanced vibration suppression algorithms in adaptive optics systems.," Journal of the Optical Society of America. A 29, 185-94 (Mar. 2012).

[3] L. Gilles, H. F. Raynaud, C. C. L. W. B. E. C. B. and Kulcs Â,r, C., "Kalman filter design for atmospheric tip/tilt, tip/tilt anisoplanatism and focus filtering on extremely large telescopes," SPIE This conference (2014).

[4] Anderson, B. D. O. and Moore, J. B., [Optimal Filtering], Dover Publications (2005).

[5] "http://model.obs-besancon.fr/."

[6] Wang, L., Ellerbroek, B., and Veran, J. P., "High fidelity sky coverage analysis via time domain adaptive optics simulations," Appl. Opt. 48, 5076-5087 (Sep 2009). 